\title{
Consumer health 2.0 in Canada: tweeting about cancer
}

\author{
Christine Marton
}

\begin{abstract}
Eight of the top ten consumer health information websites in Canada have a Twitter feed. The Twitter feed of the Canadian Cancer Society (CCS) has thousands of tweets and followers. The CCS Twitter feed utilizes many Twitter features, including retweet, hashtags, InReplyTo, URLs, and multimedia. Moreover, the CCS Twitter feed projects a positive message about conquering cancer. The majority of Twitter posts (tweets) pertain to established CCS events: Daffodil Day/Month and Relay for Life. Health promotion messages primarily concerning tobacco cessation and the avoidance of tanning regularly reoccur in CCS tweets. Cancer survivors and family members are active participants in the CCS twitter feed. Their contributions to its content is acknowledged on the CCS Twitter feed through retweets, replies (InReplyTo), and links (URLs). The CCS Twitter feed is an effective communication tool for information dissemination about cancer and is a community building tool for those whose lives have been touched by cancer.
\end{abstract}

\section{Introduction}

The Social Web or Web 2.0 has revolutionized the ways by which individuals, groups, and organizations communicate and interact with each other. User-generated content published on popular social websites facilitate information dissemination and sharing, and builds communities for those with similar interests, including health conditions. User-generated content comprises primarily textual messages with hypertext links to additional content. However, there are applications (apps) on social websites that enable users to post personal photos, videos, and audio clips, which are often embedded in textual messages.

While the use of social networking sites by individuals and grassroots groups for community building and political activism is well established, their adoption by professionals has been gradual. Librarians and clinicians have begun to utilize several Social Web tools, primarily Facebook, RSS feeds, blogs and microblogs such as Twitter, to provide information and spread awareness about their services. More importantly, a few have chosen to utilize social websites to provide online the services they traditionally offered solely within the confines of their institutions, such as reference and clinical diagnosis [1-4].

Twitter has been called a microblogging utility or platform, a short message service (SMS), a social networking site (SNS), a type of internet relay chat (IRC), and a status update service. Created in 2006 by Jack Dorsey and other members of the board of directors of the podcasting company Odeo, it was conceived of as an internal SMS for the company. Originally called twttr after the fivecharacter length of American SMS short codes, it was publicly launched later that year on 15 July 2006. Symbolized by the bluebird logo, known as Larry the Bird, Twitter is now one of the most popular social networking sites and most visited websites globally [1-6].

Twitter accounts are free. All that is required is a brief visit to the Twitter website (twitter.com), to set up a subscription with a login, password, brief bio, and an accompanying image. Twitter is easily accessible from its website, as well as by text message, instant message, short message service, and third-party platforms designed for mobile phones. Twitter owes its popularity to several unique features. Twitter has a 140-character limit for messages, referred to as tweets. This brevity is ideal for handheld computing devices. Tweets are by default publicly available; however, Twitter users can choose to make their tweets private. Twitter can accommodate hypertext links of any size embedded in tweets by automatically shortening the URLs. Twitter also supports multimedia elements such as photos and videos with embedded links; ViewPhotos and ViewVideos, respectively [1-6].

The TwitterVerse, the global Twitter community, is based on followers and followees. Not only can an individual Twitter user send messages that can be read by anyone with access to the Web, he or she can choose to follow the tweets sent by other Twitter users by becoming a follower of those Twitter feeds, while those who are interested in the content of a specific Twitter feed are referred to as its followees. The InReplyTo feature enables

Christine Marton, PhD., Adjunct Instructor. Faculty of Information, University of Toronto, 140 St. George Street, Toronto, ON, M5S 3G6.

Corresponding author (e-mail: christine.marton@utoronto.ca). 
Twitter account users to respond to specific tweets sent by others. Tweets authored by others and deemed of interest to an individual can be re-issued as retweets or tagged as favorites. Moreover, a Twitter account whose tweets are of ongoing interest to an individual Twitter user can be labelled in a tweet by "Follow Friday", which encourages other Twitter users to follow that Twitter account. Of interest to information professionals is the hashtag (\#) feature, a form of metadata that enables topics to be identified in tweets. Tagging tweets with one or more hashtags is helpful in identifying popular topics so-called trending topics. Collectively, these Twitter features facilitate message dissemination and amplification between Twitter users, and build communities of Twitter users with similar interests [1-6].

The Canadian Cancer Society (CCS) is the national notfor-profit organization of community-based volunteers in Canada dedicated to eradicating cancer and improving the quality of life of people living with cancer. The CCS provides a comprehensive consumer health information website that was ranked in 2010 as one of the top ten CHI sites in Canada by the Consumer Health Information Providers Interest Group of the Canadian Health Libraries Association [7]. In this paper, the use of the popular microblogging utility Twitter by the CCS is examined. To-date, the use of Twitter by health sciences librarians at New York University Health Sciences Library (NYUHSL) as a communications tool to inform users about its services and also as an online reference tool has been described in two recently published articles [1,2]. Detailed content analysis of tweets from individuals with dental pain, those using antibiotics, and tweets with the terms "H1N1" versus "swine flu" during the 2009 H1N1 pandemic, respectively, have appeared in the health sciences journal literature [5,6,9]. Altogether, the study of Twitter feeds is in its infancy. This study of the CCS Twitter feed is the first to analyze the content and features of a consumer healthfocused Twitter feed authored by a Canadian organization.

\section{Methods}

Visits to the Twitter feed of the CCS were conducted on Sunday, 1 April 2012 and Monday, 2 April 2012. A statistical analysis of several attributes of the CCS Twitter feed from its public launch on 6 January 2009 to 31 March 2012 was undertaken. The frequency of popular words was determined using the Find/Replace feature in Microsoft Word on the entire text of the CCS Twitter feed. A word cloud featuring the most frequently occurring words was generated. The word cloud is a visual representation of the thematic focus of the CCS Twitter feed. The total number of occurrences of key Twitter attributes - tweets, retweets, hashtags, hypertext links (URLs), InReplyTo's, Follow Fridays, ViewPhotos, and ViewVideos - were calculated for the timeframe of the CCS Twitter feed. As well, a more in-depth approach entailed calculating the frequency of the aforementioned Twitter feed attributes for each day of the month of March of 2009, 2010, 2011, and 2012. This subset of quantitative data was tabulated in Excel and the totals for all CCS Twitter feed attributes for the month of March were then entered into an SPSS data file. A line graph was generated to show the trends in frequency of occurrence of these Twitter features over the timeframe of the CCS Twitter feed. This unique statistical approach relied solely on the calculation of frequency distributions, a popular descriptive statistics tool, to provide insights concerning both the focus of the CCS Twitter feed content, and patterns of utilization of Twitter feed attributes, as detailed in the Results Section that follows.

\section{Results}

As of 1 April 2012, the CCS Twitter feed (https:// twitter.com/\#!/cancersociety) had a total of 2,109 tweets and 14,241 followers. While the number of tweets has almost doubled and the number of followers has tripled since 25 November 2010, the number of followees has slightly declined from 434 to 430 [7]. In 2009, the CCS Twitter feed had an average of 1-2 tweets per day, excluding weekends. In 2012, there is an average of 3-4 tweets per day, including weekends.

Examining the content of the entire CCS Twitter feed from 2009 to 2012, a list of frequently found words (and stems) is generated. Not surprisingly, the most frequently occurring word is cancer, with a total of 5617 occurrences in tweets. Excluding the use of this word in frequently occurring phrases, cancersociety (2410), Cdn Cancer Society (2108), Canadian Cancer Society (126), and CancerConnection [2], there are approximately 971 unique occurrences of the word cancer in the tweets of the CCS Twitter feed. The second most frequently occurring word is fight (511), commonly co-occurring with the word cancer in the text of tweets or as a hashtag. The "fight against cancer" is a well-known CCS phrase in all of its media outlets. The third most frequently occurring word in the CCS Twitter feed is daffodil (372), the symbol of the CCS. It is frequently found in tweets as a hashtag, \#daffodilday (28) and \#daffodilmonth (134). The fourth and fifth most frequently found words are life (251) and relay (243). These two words frequently co-occur as a hashtag \#relayforlife, which is a popular CCS annual event. Other popular terms are support (191), help (171), health (142), research (114), smok (98), event (89), breast (85), and story (84). The number of word occurrences detailed above may include over-estimates and under-estimates, as some Microsoft Word Find/Replace searches on these words do not take into consideration the possibility that they can be found within other words, e.g. story is situated within history, or that they can function as roots or stems in truncation, e.g. research also includes researcher and researchers, and smok includes smoke, smoking, smoker, and smokers. In Figure 1 on the next page, words that have over 100 occurrences in the CCS Twitter feed are presented as a word cloud, with each word's frequency represented by font size. The frequently occurring words present a positive and proactive approach to a grim disease; the words health, life, help, fight, support, and research collectively suggest that cancer can be beaten with the right tools. Equally of interest is the absence or small number of occurrences of negative words commonly associated with cancer: neoplasm (0), tumor (0), tumour (4), chemo (4), surgery (0), radiation (6), disease (13), and death (23). 
Fig. 1. Word cloud of frequently occurring terms in CCS tweets.

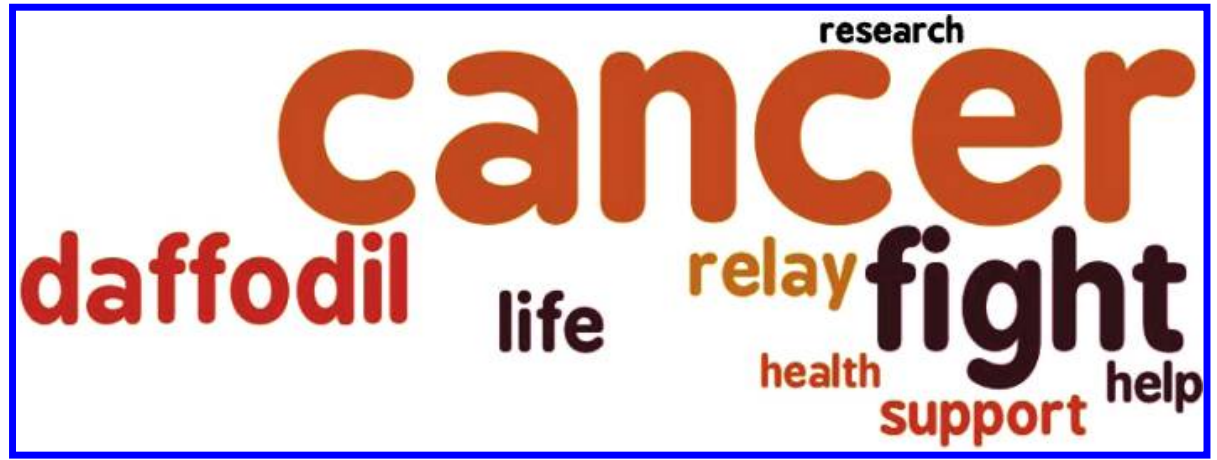

The different types of cancer are also underplayed, with the exception of breast (85), which is linked to health promotion messages about breast screening and mammograms (25), and an event, thingmaboob (32). Word occurrences for common types of cancer are lung (24), colon (22), prostate (22), skin (24), while other types of cancer merit less than 10 word occurrences. It seems plausible that there is a deliberate avoidance of negative terms in the tweets from the CCS twitter feed. Of interest to information professionals, there are 36 occurrences of the word "information", 16 occurrences of the word "resource", and nine occurrences of the cancer helpline phone number, 1-888-939-3333, in CCS tweets.

The frequency of use of popular Twitter features over the time span of the CCS Twitter feed was also examined. There are 667 occurrences of ReTweet (RT), 747 hashtags, 332 occurrences of InReplyTo, 91 occurrences of ViewPhoto, and 32 occurrences of ViewVideo, and 23 occurrences of FollowFriday. These numbers are approximations for several reasons; the hashtag symbol (\#) may be used to symbolize something else, e.g. \#1, or may not be used consistently in tweets, e.g. twibbon and \#twibbon, while photos and videos can be included in tweets without using the ViewPhoto and ViewVideo features. The most frequently used URL shortening services in the CSS Twitter feed are ow.ly (726), followed by bit.ly (201), tinyURL (76), t.co (7), and twb.ly (7).

The pattern of use of Twitter features in the CCS Twitter feed was examined more closely by extracting the daily number of occurrences of each feature for a fixed period of time over the time span of the CCS Twitter feed; the month of March in 2009, 2010, 2011, 2012. In March 2009, the pattern of use of Twitter features was quite rudimentary, with a total number of 25 tweets in that month. There were no occurrences of retweets, hashtags, InReplyTo, ViewPhoto, ViewVideo, and FollowFriday. However, there were 24 URLs, and one link to a photo (18 March), and one link to a video (31 March). There were no tweets on weekends. The highest number of tweets per day was two tweets (4, 6, 19, 26 March), with one tweet per weekday as the norm. Many tweets contained a URL. Clearly, the first year of the CCS Twitter feed was marked by a small number of tweets and very limited use of Twitter features. However, the tweets extracted from the CCS Twitter feed from March 2010 show a marked increase in the number of daily tweets and the use of Twitter features. There was a total of 105 tweets, of which 45 were retweets. The 45 tweets contained 76 URLs, 6 InReplyTo, 21 hashtags, 8 ViewPhoto, 4 ViewVideo, and 1 FollowFriday. The highest number of tweets was 13 (25 March) and the highest number of retweets was 8 (25 March). More tweets were posted in the second half of the month. There were no tweets on weekends. Most tweets contained at least one URL. Clearly, the level of sophistication of Twitter use had improved significantly. In subsequent years, CCS tweets from the month of March have been characterized by a large number of tweets, retweets and URLs, but not as many as March 2010. However, the number of hashtags has grown impressively, with 52 hashtags in March 2012. Table 1 summarizes the CCS Twitter feed data from the month of March.

A graphical representation of this quantitative data in Figure 2 demonstrates that the number of tweets and retweets and URLs peaked in 2010 and have since plateaued or declined, while the number of hashtags grew sharply in 2012.

The final subset of data extracted from the CCS Twitter feed are printouts of the tweets from the days with the highest number of tweets in the timeframe of the CCS Twitter feed: 4 June 2010; 11 June 2010; 10 September 2010; 1 April 2011; 7 April 2011; 18 April 2011, and 12 September 2011. The high tweet frequency days are event-driven. On 4 June 2010, there was a total number of 28 tweets of which 9 are retweets. There were 21 hashtags, 11 occurrences of InReplyTo, and 5 occurrences of ViewPhoto. The reoccurring hashtag \#relayforlife reveals the predominant theme, the annual CCS Relay for Life marathon. Similarly, on 11 June 2010, there were a total of 21 tweets, of which 12 were retweets. There were 14 hashtags, 4 occurrences of InReplyTo, and 8 occurrences of ViewPhoto. The tweets revolved around the hashtag \#relayforlife. On 10 September 2010, there was a total of 33 tweets, of which 10 were retweets. There were 22 hashtags, but 0 occurrences of ViewPhoto. The reoccurring hashtag \#fightcancer was frequently embedded in tweets concerning the CityTV event, City Fights Cancer. On 13 April 2011, there was a total number of 29 tweets, with 19 retweets. There were 20 hashtags and 4 occurrences of ViewPhoto. April is the annual CCS sale of daffodils, as denoted by the messages, "April is the month to fight 
Table 1. Total number of occurrences of Twitter features in the CCS Twitter feed, month of March.

\begin{tabular}{|c|c|c|c|c|c|c|c|c|}
\hline Year & Tweets & Retweets & URL & InReplyTo & Hashtag & ViewPhoto & ViewVideo & FollowFriday \\
\hline 2010 & 105 & 45 & 76 & 6 & 21 & 8 & 4 & 1 \\
\hline 2012 & 93 & 23 & 56 & 13 & 52 & 6 & 5 & 1 \\
\hline
\end{tabular}

Fig. 2. The frequency of tweets, retweets, URLs and hashtags in the CCS Twitter feed in March.

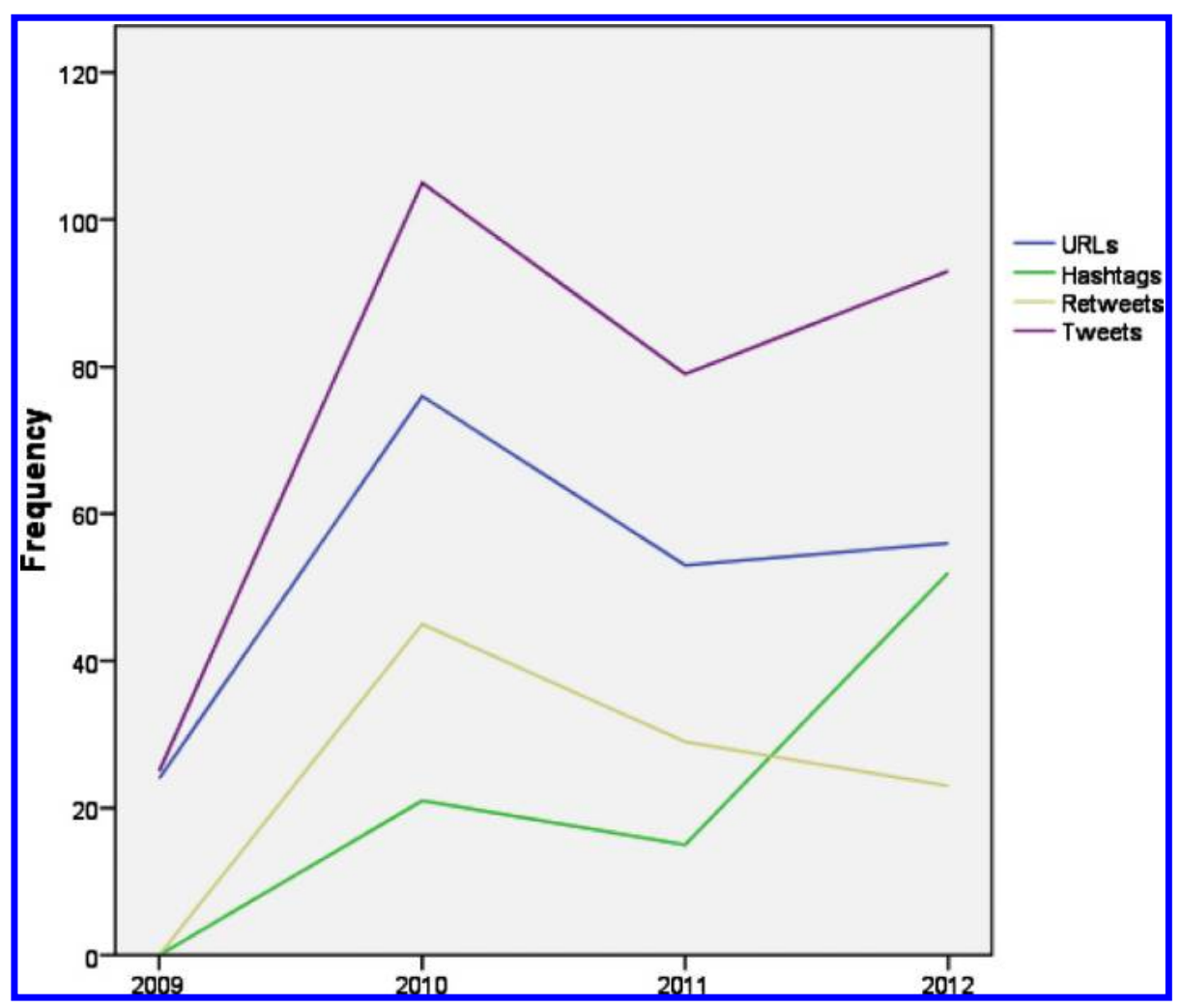

back" and "Who are you fighting for this April"? The hashtags \#DaffodilDay and \#DaffodilMonth dominate CCS tweets. On 7 April and 18 April 2011 there were a large number of tweets, 28 and 31 respectively, for the same CCS event. On 12 September 2011, it was rather unique because the CCS Twitter feed had the highest number of tweets, with 41 tweets but 0 retweets. There were no occurrences of Twitter features, InReplyTo, ViewPhoto, and ViewVideo. However, there were 87 hashtags, primarily \#voteON and \#LNCdebate, which frequently co-occurred in tweets, as well as the twitter account handle of the Ontario Minister of Health and Long-Term Care, Deb Matthews (@Deb_Matthews). The lack of interactivity in the CCS twitter feed on this day is a marked departure from other days with large numbers of tweets, all of which promoted cancer events. The focus of the CCS tweets on 11 September 2011, is the dissemination of the governing Ontario Liberal Party's health promotion policies, and those of the Green Party candidate Kevin Labonte (@KevinLabonte), particularly as they pertain to cancer prevention. Clearly, the CCS utilized its Twitter feed to encourage its members to influence provincial health policy by voting for political parties who have a health promotion mandate.

\section{Discussion}

The Twitter feed of the CCS has shown an increase in the number of tweets and use of Twitter features over time. There is a greater sophistication in the use of the metadata feature, hashtags in recent years, but inconsistencies persist. As well, the use of multimedia features such as ViewPhoto and ViewVideo remains modest, and other Twitter features, most notably, FollowFriday, are rarely utilized.

Word counts of the CCS Twitter feed across its almost 4-year lifespan demonstrate a positive message about cancer; that with research and support, cancer can be beaten. The majority of tweets pertain to established CCS events, Daffodil Day/Month and Relay for Life and feature the participation of its membership in its events and their reasons for participation. However, there are surprisingly 
few tweets about the CCS lottery. Health promotion messages pertaining to breast cancer screening, smoking cessation, the risks of tanning beds, are also present in the CCS Twitter feed. Cancer survivors and family members are active participants in the CCS twitter feed, and their contributions to its content are acknowledged by the CCS through retweets, replies (InReplyTo), and links (URLs). The CCS Twitter feed is an effective communication tool for information dissemination about cancer and community building for those whose lives have been touched by cancer.

\section{References}

1. Cuddy C. Twittering in health sciences libraries. Journal of Electronic Resources in Medical Libraries. 2009;6(2):169-73. doi:10.1080/15424060902932284.

2. Cuddy C, Graham J, Morton-Owens EG. Implementing twitter in a health sciences library. Med Ref Serv $Q$. 2010;29(4):320-30. doi:10.1080/02763869.2010.518915.

3. DeFebbo DM, Mihlrad L, Strong MA. Microblogging for medical libraries and librarians. Journal of Electronic Resources in Medical Libraries. 2009;6(3):211-23. doi:10.1080/ 15424060903167385.
4. Hawn C. Report from the field: Take two aspirin and tweet me in the morning: How twitter, facebook, and other social media are reshaping health care. Health Aff. 2009;28(2):361-8. doi:10.1377/hlthaff.28.2.361.

-5. Heaivilin N, Gerbert B, Page JE, Gibbs JL. Public health surveillance of dental pain via twitter. $J$ Dent Res. 2011;90(9):1047-51. doi:10.1177/0022034511415273.

-6. Scanfeld D, Scanfeld V, Larson EL. Dissemination of health information through social networks: Twitter and antibiotics. Am J Infect Control. 2010;38(3):182-8. doi:10.1016/j.ajic. 2009.11.004.

7. Consumer Health Information Providers Interest Group. Top 10 Canadian consumer health websites. 2010. [cited 2012 April 1]; Available: http://www.chla-absc.ca/chipig/Events/ CHLA2010poster.pdf.

8. Marton C. Consumer health 2.0 in Canada: a descriptive analysis of the use of Web 2.0 technologies on Canadian consumer health information websites, JCHLA 2010: 32(1), 29-34. doi:10.5596/c11-009.

9. Chew C, Eysenbach G. Pandemics in the age of Twitter: content analysis of Tweets during the 2009 H1N1 outbreak. PLoS One. 2010: 5(11), e14118. doi:10.1371/journal.pone. 0014118. 